\title{
Overview of Global Neutron Sources, Instruments and Initiatives
}

\author{
Ken Andersen \\ Oak Ridge National Laboratory, Oak Ridge, Tennessee, United States of America; \\ andersenkh@ornl.gov
}

Neutron scattering is an essential element in the materials science toolkit, providing unique structural and dynamic information. It relies on an ecosystem of facilities and smaller sources, which provide access to researchers covering a vast range of scientific problems. This talk will provide an overview of the current global neutron landscape, both today and in the near future. I aim to demonstrate the scientific impact, diversity and vitality of this ecosystem, highlighting the important role that neutron scattering plays in addressing a number of societally-impactful grand challenges.

Keywords: Neutron scattering, user facilities 